\title{
4-Hydroxybenzaldehyde Restricts the Intracellular Growth of Toxoplasma gondii by Inducing SIRT1-Mediated Autophagy in Macrophages
}

\author{
Jina Lee ${ }^{1,2}$, Jae-Won Choi ${ }^{1,2}$, Hye Young Han', Woo Sik Kim³, Ha-Yeon Song ${ }^{3}$, Eui-Baek Byun ${ }^{3}$, Eui-Hong Byun ${ }^{4}$, \\ Young-Ha Lee', Jae-Min Yuk ${ }^{1,2 *}$ \\ 'Department of Infection Biology and Medical Science, College of Medicine, Chungnam National University, Daejeon 35015, Korea; ${ }^{2}$ Infection \\ Control Convergence Research Center, College of Medicine, Chungnam National University, Daejeon 35015, Korea; ${ }^{3}$ Advanced Radiation \\ Technology Institute, Korea Atomic Energy Research Institute, Jeongeup 56212, Korea; ${ }^{4}$ Department of Food Science and Technology, Kongju \\ National University, Yesan 32439, Korea
}

\begin{abstract}
Toxoplasma gondii is an intracellular protozoan parasite that infects approximately one third of the human population worldwide. Considering the toxicity and side effects of anti-toxoplasma medications, it is important to develop effective drug alternatives with fewer and less severe off-target effects. In this study, we found that 4-hydroxybenzaldehyde (4HBA) induced autophagy and the expression of NAD-dependent protein deacetylase sirtuin-1 (SIRT1) in primary murine bone marrow-derived macrophages (BMDMs). Interestingly, treatment of BMDMs with 4-HBA significantly reduced the number of macrophages infected with $T$. gondii and the proliferation of $T$. gondii in infected cells. This effect was impaired by pretreating the macrophages with 3-methyladenine or wortmannin (selective autophagy inhibitors) or with sirtinol or EX527 (SIRT1 inhibitors). Moreover, we found that pharmacological inhibition of SIRT1 prevented 4-HBA-mediated expression of LC3-phosphatidylethanolamine conjugate (LC3-II) and the colocalization of T. gondii parasitophorous vacuoles with autophagosomes in BMDMs. These data suggest that 4-HBA promotes antiparasitic host responses by activating SIRT1mediated autophagy, and 4-HBA might be a promising therapeutic alternative for the treatment of toxoplasmosis.
\end{abstract}

Key words: Toxoplasma gondii, toxoplasmosis, autophagy, sirtinol, 4-hydroxybenzaldehyde, NAD-dependent protein deacetylase sirtuin-1, LC3-phosphatidylethanolamine conjugate

\section{INTRODUCTION}

Toxoplasma gondii, a member of the Apicomplexa phylum, is an obligate protozoan pathogen that can invade and replicate in almost all nucleated cells of warm-blooded animals. Almost a third of the global human population is infected with T. gondii $[1,2]$. For most immunocompetent individuals, infection is asymptomatic, chronic, and life-long; however, T. gondii infection in immunocompromised individuals and pregnant women can cause severe illness with high morbidity and mortality rates $[2,3]$. First-line treatment for toxoplasmosis is combined administration of pyrimethamine and sulfadiazine; however, because of their potential off-target effects, there is an

- Received 2 September 2019, revised 21 October 2019, accepted 4 November 2019.

*Corresponding author (yjaemin0@cnu.ac.kr)

(c) 2020, Korean Society for Parasitology and Tropical Medicine

This is an Open Access article distributed under the terms of the Creative Commons Attribution Non-Commercial License (https://creativecommons.org/licenses/by-nc/4.0) which permits unrestricted non-commercial use, distribution, and reproduction in any

medium, provided the original work is properly cited. urgent need to develop therapeutic alternatives with fewer, more benign side effects [4].

Autophagy, which plays a pivotal role in maintaining cellular homeostasis, is a mechanism by which cells remove dysfunctional or dispensable cellular components such as damaged cytosolic organelles and long-lived/misfolded proteins through the fusion of autophagosomes and lysosomes $[5,6]$. Accumulating evidence suggests that selective autophagy, also known as xenophagy, helps protect the host against diverse infectious agents including bacteria, viruses, and protozoa $[7,8]$. During T. gondii infection, the host's autophagy machinery contributes to the restriction of intracellular survival by maintaining a balance between the host immune response and exploitation of the host by the parasite [9].

Prior studies of 4-hydroxybenzaldehyde (4-HBA), an essential bioactive constituent of the traditional Chinese herb Gastrodiae Rhizoma, reported anti-diabetic properties [10], antioxidant properties [11], and angiogenesis-promoting effects [12]. We previously reported that 4-HBA protects against DSS-in- 
duced colitis by regulating immune tolerance and excessive inflammatory responses [13]. A recent study demonstrated that 4-HBA increased the susceptibility to amphenicol antibiotics including chloramphenicol and thiamphenicol in Acinetobacter baumannii [14]; however, the function of 4-HBA in infectious diseases has not yet been characterized.

In this study, we investigated the immunomodulatory properties of 4-HBA in T. gondii-infected murine bone marrow-derived macrophages (BMDMs). We found that 4-HBA restricts the intracellular growth of T. gondii via SIRT1-mediated autophagy activation. Collectively, our results suggest that 4-HBA may be a promising therapeutic alternative to treat patients infected with T. gondii.

\section{MATERIALS AND METHODS}

\section{Mice and cell culture}

Wild-type C57BL/6 mice were purchased from Koatech (Gyeonggi-do, Korea). Animal-related experimental procedures were approved by the Institutional Animal Care and Use Committee, Chungnam National University College of Medicine (CNU-00706; Daejeon, Republic of Korea). Bone marrowderived macrophages were differentiated during 5-7 days in medium with macrophage colony-stimulating factor, as described previously [15]. The culture medium is consisted of Dulbecco's modified Eagle's medium (DMEM; Welgene) supplemented with $10 \%$ fetal bovine serum (FBS, Gibco BRL) and 1\% Antibiotic-Antimycotic (Gibco ${ }^{\mathrm{TM}}$ Antibiotic-Antimycotic (100X); Gibco BRL). The human retinal pigment epithelial cell line ARPE-19 (American Type Culture Collection, Manassas, Virginia, USA) were grown in DMEM/F-12 (Welgene) with $10 \%$ FBS and 1\% Antibiotic-Antimycotic.

\section{Parasite preparation}

T. gondii RH strain was multiplied in ARPE-19 cells at a multiplicity of infection (MOI) of 5 and grown for 2-3 days at $37^{\circ} \mathrm{C}$ and $5 \% \mathrm{CO}_{2}$. T. gondii $\mathrm{RH}$ strain expressing transgenic green fluorescent protein (GFP-RH) were kindly provided by Dr. Yoshifumi Nishikawa (Obihiro University of Agriculture and Veterinary Medicine, Japan). Host cell debris and parasites were washed in phosphate-buffered saline (PBS) after spontaneous host cell rupture. Final pellet was suspended in cold DMEM, and then passed through a 26-gauge needle and a 5.0 $\mu \mathrm{m}$ pore filter (Millipore, Billerica, Massachusetts, USA).

\section{Reagents and antibodies}

4-HBA (144088), 3-methyladenine (3-MA, M9281), EX-527 (E7034), sirtinol (S7942), wortmannin (WM, W1628), dimethylsulfoxide (DMSO, D2650) or LC3 (L8918) were from Sigma-Aldrich. $\beta$-Tubulin (ab6046) and SIRT1 (AB28170) were purchased from Abcam. Ethanol or DMSO was added to macrophages cultures at $0.05 \%(\mathrm{v} / \mathrm{v})$ and used as a solvent control.

\section{Cell viability assays}

The cytotoxicity effects of 4-HBA on BMDMs were determined using cell count kit (CCK) 8 (Dojindo Molecular Technologies), according to the manufacturer's instructions. CCK8 solution $(10 \mu \mathrm{l})$ was added after cells were incubated with 4-HBA for 18 or $48 \mathrm{hr}$. Absorbance was measured at $450 \mathrm{~nm}$ on a microplate reader (SpectraMax ABS Plus, Molecular Devices).

RNA extraction, real-time quantitative PCR, and western blot analysis

RNA extraction and real-time quantitative PCR were performed as described previously [15]. The sequences of the primers used were as follows: Sag1 (forward: 5'-ATCGCCTGAGAAGCATCACT-3'; reverse: 5'-GCGAAAATGGAAACGTGACT-3'), $\beta$-actin (forward: 5'-TCATGAAGTGTGACGTTGACATCCGT-3'; reverse: 5'-CCTAGAAGCATTTGCGGTGCACGATG-3').

Western blot analysis was performed as described previously [15]. Collected cell lysates was lysed using RIPA buffer (10 mM Tris-HCl at pH 8.0, 1 mM EDTA, $140 \mathrm{mM} \mathrm{NaCl}, 0.1 \%$ SDS, $0.1 \%$ sodium deoxycholate and $1 \%$ Triton X-100) containing a protease inhibitor cocktail (Roche). The protein extracts boiled with SDS sample buffer was subjected to sodium dodecyl sulfate polyacrylamide gel electrophoresis and then polyvinylidene fluoride membranes (Millipore Corp.). Chemiluminescence assay kit (ECL; Millipore Corp.) was used to develope membranes.

\section{Quantification of intracellular T. gondii}

BMDMs cultured on 22-mm glass coverslips were infected with GFP-RH strain for indicated time periods, and then coverslips were washed using warmed PBS. After fixation with $4 \%$ paraformaldehyde in PBS for 10 min and permeabilization with 0.25\% Triton X-100 in PBS for 10 min, Texas Red ${ }^{\circledR}$-X phalloidin (Life Technologies Corporation) and 4'6-diamidino-2-phenylindole (DAPI, Sigma) were used to visualize F-actin in the cytoskeleton and nucleus. Cover slides were analyzed by confocal laser scanning microscopy (Leica TCS SP8, Leica microsystems). 
Immunofluorescence analyses

Immunofluorescence analysis of endogenous LC3 puncta and colocalization of LC3 with GFP-RH was performed as described previously [16]. Followed by fixation and permeabilization, cells in coverslips were stained with LC3 Ab (MBL International, PM036) for $2 \mathrm{hr}$ at room temperature. After washing excess primary $\mathrm{Ab}$ with $\mathrm{PBS}$, cells were incubated with $\mathrm{Al}-$ exa Fluor 488-conjugated goat anti-rabbit IgG (Molecular Probes) or Alexa Fluor 594-conjugated goat anti-rabbit IgG (Molecular Probes) for $2 \mathrm{hr}$. Nucleus was stained with DAPI. Fluorescence images were obtained using confocal laser scanning microscopy. Each experiment for immunofluorescence microscopy contains at least 70 cells scored from 8 random fields for analysis of endogenous LC3 puncta. 70 internalized GFP-RH per experimental condition were counted for quantitative data of colocalization between LC3 with GFP-RH.

\section{Statistical analysis}

A 2-tailed Student's $t$ test was used to analyze differences between independent experimental data (means \pm standard deviation [SD] or means \pm standard error [SEM]). Differences were deemed significant at $P$-value under 0.05 .

\section{RESULTS}

\section{Cytotoxic effects of 4-HBA in BMDMs}

To evaluate the cytotoxicity of 4-HBA, we assessed the effects of 4-HBA on BMDM viability by CCK8 assay. Incubating BMDMs with $10 \mu \mathrm{M}$ 4-HBA had no significant effect on cell viability after $18 \mathrm{hr}$ (Fig. 1A) or $48 \mathrm{hr}$ (Fig. 1B); however, cell viability decreased by approximately $35 \%$ at $24 \mathrm{hr}$ (Fig. 1A) and $92 \%$ at 48 hr after treatment with $50 \mu \mathrm{M}$ 4-HBA (Fig. 1B). Based on these results, for subsequent experiments we used $10 \mu \mathrm{M}$ 4-HBA, a concentration that did not induce cytotoxicity in BMDMs, and evaluated the antiparasitic effects of 4-HBA on T. gondii infection.

\section{4-HBA inhibitsed the intracellular growth of $T$. gondii in BMDMs}

T. gondii can invade and live within all nucleated cells (including macrophages and dendritic cells) by forming parasitophorous vacuoles (PVs) [17], thus, we tested whether 4-HBA exhibit antiparasitic effects against $T$. gondii infection in BMDMs. BMDMs were infected with a GFP-expressing RH strain of T. gondii (GFP-RH) for the indicated periods (Fig. 2A-C), and then evaluated for intracellular growth of $T$. gondii. As shown in Fig. $2 \mathrm{~A}$ and $\mathrm{B}$, the number of T. gondii-infected cells was markedly suppressed by 4-HBA treatment in a dose-dependent manner. Moreover, intracellular proliferation of the parasite was also inhibited in 4-HBA-stimulated BMDMs when compared to BMDMs treated with SC (Fig. 2A and C). In addition, we assessed the antiparasitic effects of 4-HBA by analyzing the expression levels of T. gondii surface antigen 1 (SAG1). As shown in Fig. 2D, SAG1 mRNA expression significantly decreased in 4-HBA-treated BMDMs in a concentration-dependent manner. These results indicate that 4-HBA plays an essential role in the activation of antiparasitic responses to T. gondii infection.

4-HBA-induced autophagy played an essential role in the activation of antiparasitic responses in primary murine macrophages

Previous studies demonstrated that autophagy contributes to host immune defenses against T. gondii infection $[8,18]$; however, the role of 4-HBA in autophagy activation has not been characterized. To determine whether 4-HBA treatment
A

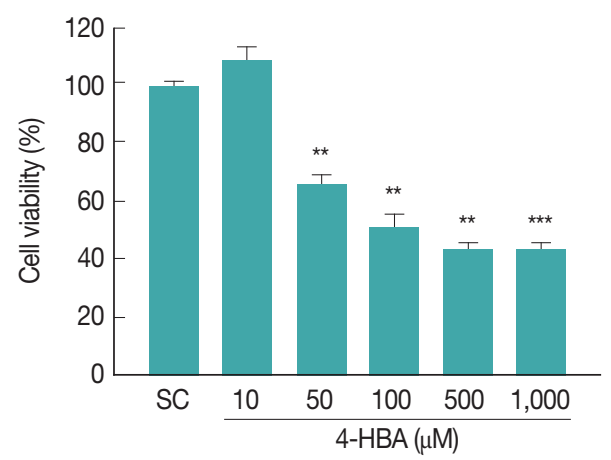

B

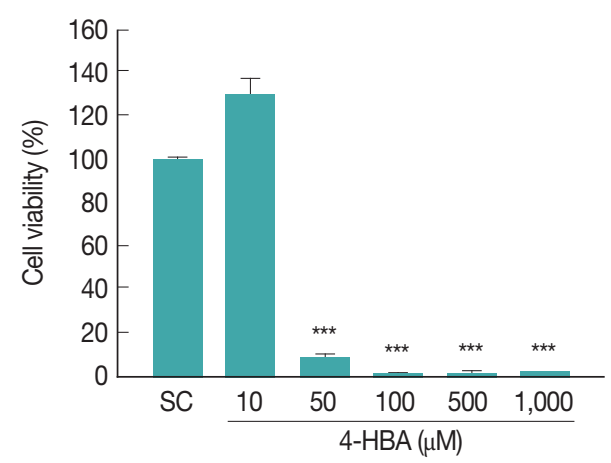

Fig. 1. Cytotoxic effect of 4-HBA on BMDMs by CCK8 assays. (A, B) Evaluation of cell viability at $18 \mathrm{hr}$ (A) or $48 \mathrm{hr}(\mathrm{B})$ after 4-HBA treatment in BMDMs. ${ }^{* \star} P<0.01,{ }^{\star \star \star} P<0.001$. SC, solvent control. 

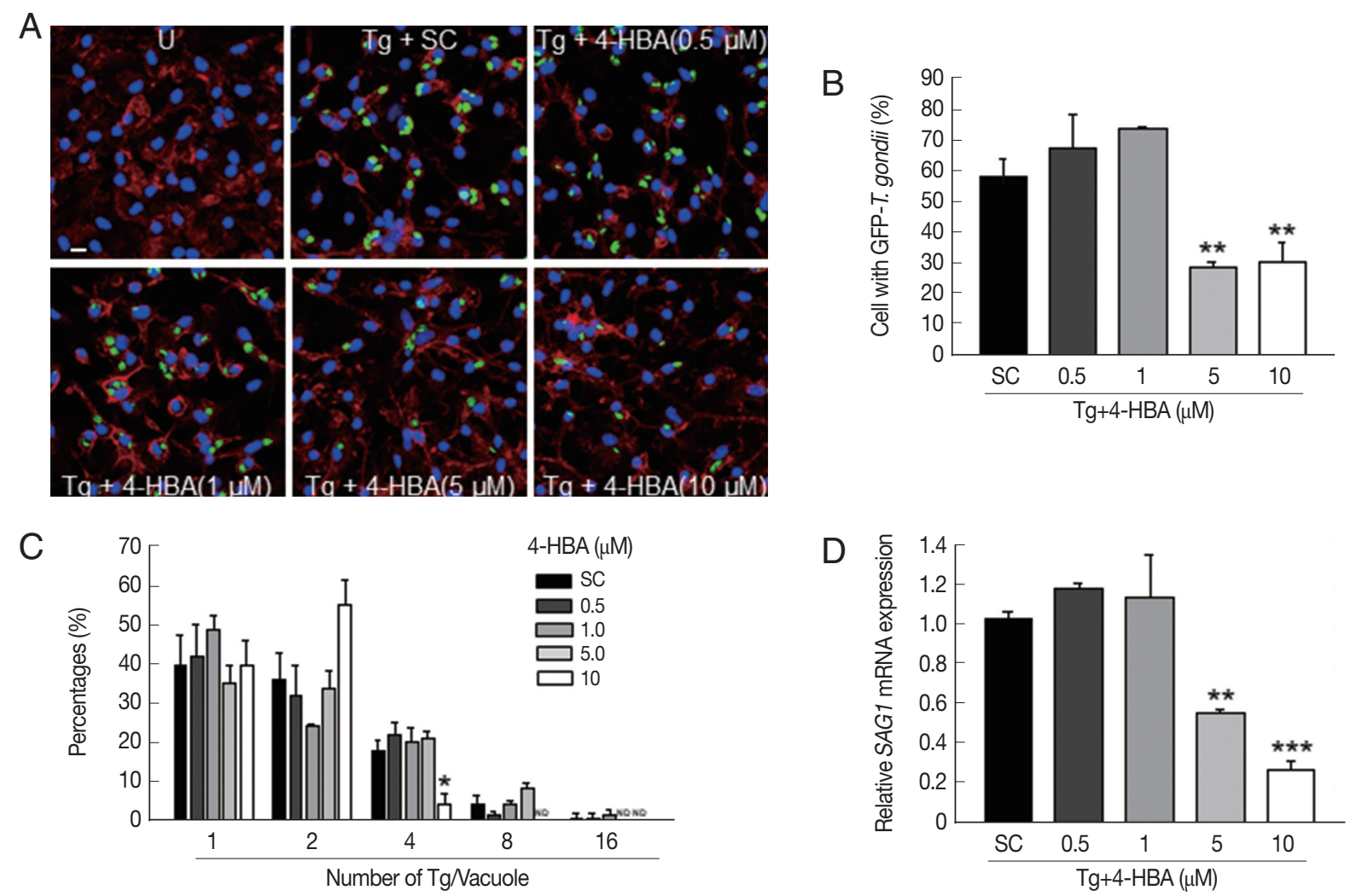

Fig. 2. 4-HBA increased antiparasitic activity in T. gondii-infected macrophages. (A-D) BMDMs were infected with GFP-RH strain (A, B, and $\mathrm{C}$ ) or $\mathrm{RH}$ strain (D) for $2 \mathrm{hr}(\mathrm{moi}=1)$ and then treated with 4-HBA for $18 \mathrm{hr}$. (A) Representative images showing T. gondii invasion into and growth in BMDMs. Scale bar $=10 \mu \mathrm{m}$. (B) Proportion of cells infected with T. gondii to total cells. (C) Number of parasites per vacuole. (D) Quantitative r-t PCR on SAG1 mRNA. ${ }^{\star} P<0.05,{ }^{\star \star} P<0.01,{ }^{\star \star \star} P<0.001$, U, untreated; SC, solvent control; Tg, T. gondii; ND, not detected.

induces autophagy in macrophages, we evaluated the number of cells with microtubule-associated protein 1 light chain 3 (LC3), an essential autophagy effector. As shown in Fig. 3A, 4-HBA treatment increased the number of LC3 aggregates in BMDMs. We next attempted to investigate the role of autophagy in 4-HBA-mediated anti-toxoplasma activity. As shown in Fig. 3B, the anti-toxoplasma activity of 4-HBA was significantly attenuated by treating T. gondii-infected BMDMs with autophagy inhibitors (3MA or WM). Our results suggest that autophagy is required for 4-HBA-induced activation of antiparasitic responses in primary macrophages.

\section{4-HBA activatesd the induction of SIRT1, which contributes to the activation of antiparasitic responses in primary murine macrophages}

Growing evidence suggests that SIRT1 is an essential regulator of diverse physiological conditions and activities such as cancer, metabolism, angiogenesis, and neuronal development
$[19,20]$. Moreover, recent studies demonstrate that SIRT1 can regulate innate and adaptive immune responses, and impaired SIRT1 activity is closely linked with the pathogenesis of autoimmune and inflammatory diseases [21]. We found that 4-HBA increased the expression of SIRT1 within $2 \mathrm{hr}$, and the increase was sustained for up to $18 \mathrm{hr}$ in BMDMs (Fig. 4A). Next, we investigated if SIRT1 was required for 4-HBA-induced antiparasitic responses. SAG1 mRNA expression decreased significantly in 4-HBA-treated BMDMs (Fig. 4B and C); however, these effects disappeared in the presence of EX-527 (Fig. 4B) or sirtinol (Fig. 4C), selective SIRT1 inhibitors. These data suggest that SIRT1 controls 4-HBA-mediated growth inhibition of T. gondii in primary murine macrophages.

\section{SIRT1 is required for 4-HBA-mediated activation of autophagy in BMDMs}

Previous studies demonstrate that SIRT1 regulates autophagy activation through the deacetylation of autophagy-related 
A
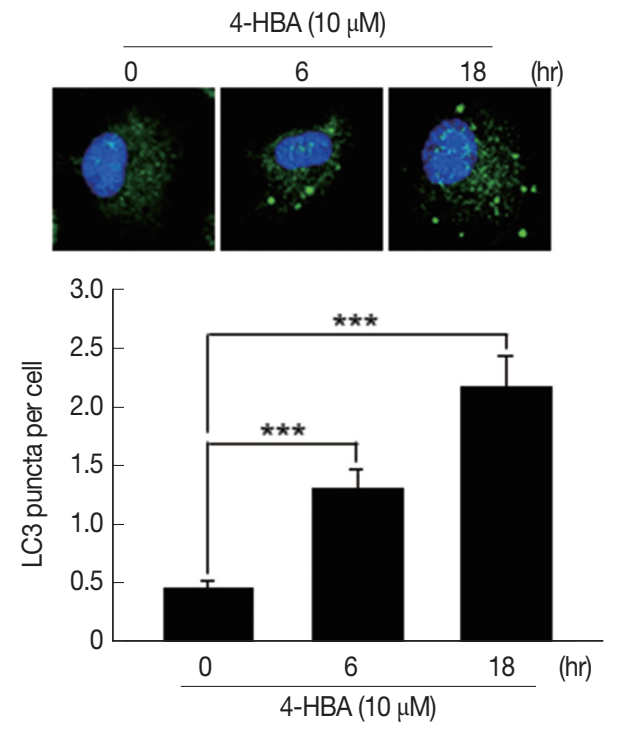

B

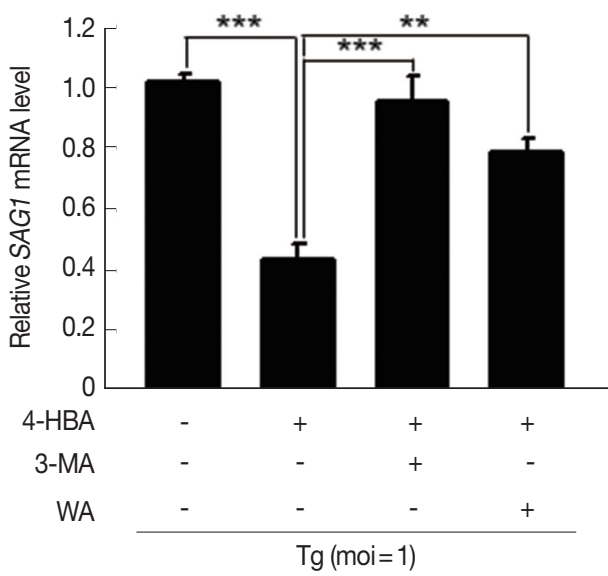

Fig. 3. 4-HBA-induced autophagy was required for the intracellular restriction of T. gondii in BMDMs. (A) Immunofluorescence microscopy on LC3 puncta formation in BMDMs treated with 4-HBA. Representative images (top; Scale bar=10 $\mu \mathrm{m}$ ). LC3 punctate foci per cell (Lower panel). (B) SAG1 mRNA level guantifed by rt-PCR. BMDMs were infected with T. gondii RH strain, treated with 3-MA (10 $\mu \mathrm{M})$ or WM $(100 \mathrm{nM})$, and then incubated with 4-HBA $(10 \mu \mathrm{M})$ for $18 \mathrm{hr} .{ }^{\star \star} P<0.01,{ }^{\star \star \star} P<0.001$.

A

4-HBA $(10 \mu \mathrm{M})$

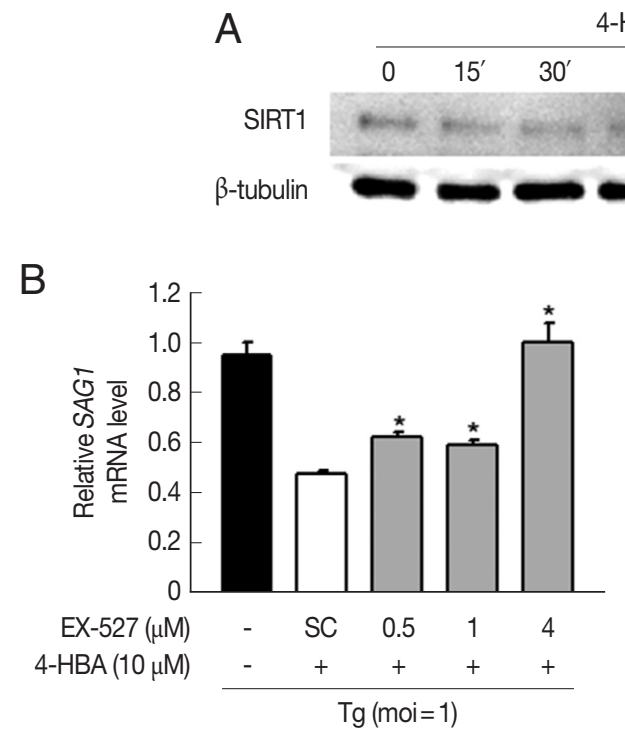

C

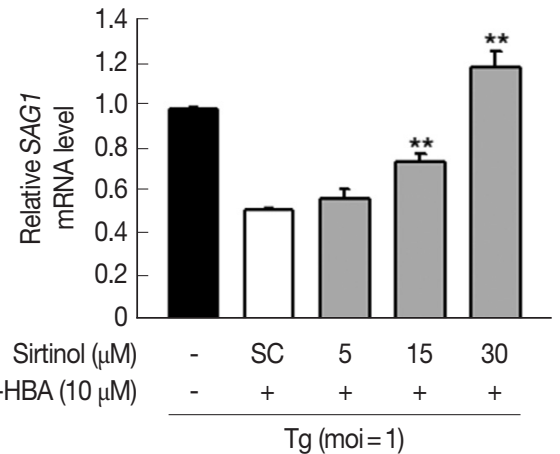

Fig. 4. Sirt1 was involved in 4-HBA-mediated activation of antiparasitic responses in BMDMs. (A) Immunoblot of SIRT1 and $\beta$-tubulin in BMDMs treated with 4-HBA. (B, C) SAG1 mRNA level. BMDMs were infected with RH strain in the presence of EX-527 (B) or sirtinol (C) and incubated with 4-HBA for $18 \mathrm{hr} .{ }^{*} P<0.05,{ }^{* *} P<0.01$. SC, solvent control; Tg, T. gondii.

genes (Atgs) and regulators of autophagy [22]. To examine whether 4-HBA-induced autophagy is mediated by SIRT1, we evaluated the formation of autophagosomes by BMDMs. We found that 4-HBA-mediated the increase of LC3-positive vesicles was significantly attenuated by pretreating BMDMs with EX-527 or sirtinol (Fig. 5A). Moreover, we also found that 4-HBA significantly increased the intracellular colocalization of LC3-II-positive autophagic vacuoles with T. gondii-containing PVs in BMDMs as compared to non-treated infected cells; however, these colocalizations were significantly attenuated by EX-527 or sirtinol (Fig. 5B, C). These data suggest that SIRT1 is an essential mediator in 4-HBA-induced autophagy activation in primary macrophages. 

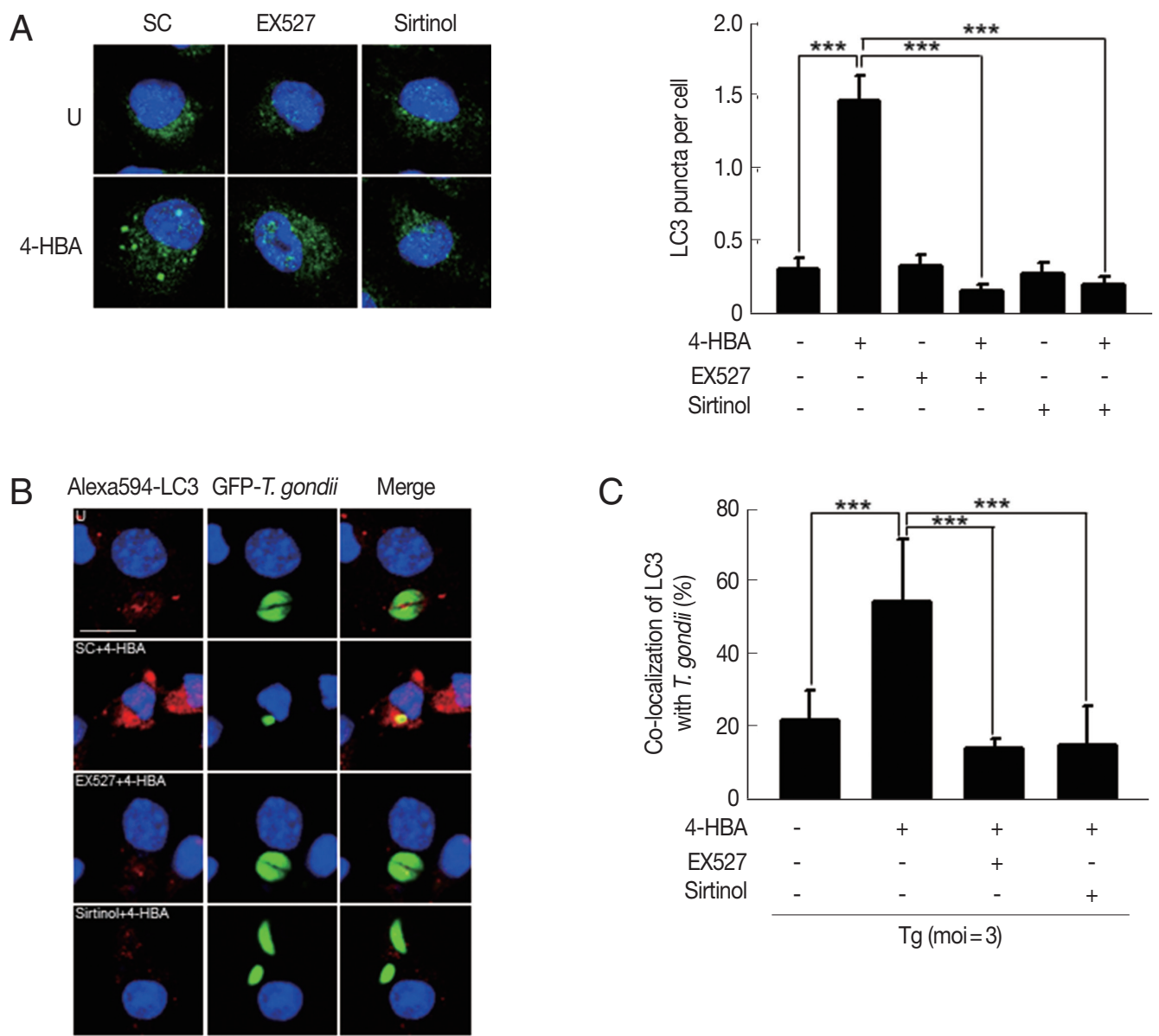

Fig. 5. Sirt1 contributed 4-HBA to induce formation and maturation of autophagosome and led activation of antiparasitic responses to $T$. gondii infection. (A) Immunofluorescence micrographs showing the formation of LC3 puncta in BMDMs treated with 4-HBA (10 $\mu$ M;

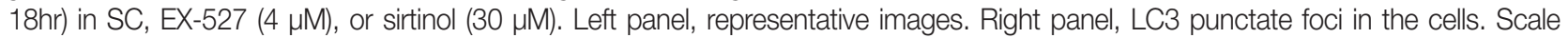
bar $=10 \mu \mathrm{m}$. (B, C) BMDMs were infected with GFP-RH strain, followed by treatments of SC, EX-527 $(4 \mu \mathrm{M})$, or sirtinol $(30 \mu \mathrm{M})$, and then incubated with 4-HBA $(10 \mu \mathrm{M})$ for 18 hr. Cells were stained with Alexa 594-conjugated LC3 (red) and DAPI (blue), (B) Immunofluorescence micrographs showing co-localization of GFP-RH strain (green) and LC3 (red). Scale bar $=10 \mu$ m. (C) Co-locol of LC3 with $T$. gondii. ${ }^{* \star \star *} P<0.001$. SC, solvent control; Tg, T. gondii.

\section{DISCUSSION}

Autophagy is a tightly regulated homeostatic process that is essential to maintain cellular integrity, and impaired autophagy activation has been linked to the progression of physiological and pathological disorders [23]. Accumulating evidence suggests that xenophagy, a specialized, selective form of autophagy, is involved in activating protective host immunity responses via canonical and non-canonical pathways [24]; thereby, eliminating intracellular pathogens $[7,25]$. Many studies have evaluated flavonoids, a group of plant metabolites, as potential autophagy-inducing agents, which may be promising therapeutic alternatives for various autophagy de- regulation-related diseases [26]. During T. gondii infection, autophagy can either protect the host or promote the pathophysiology of the parasite. Initial studies demonstrate that $T$. gondii promotes autophagy in host cells so they may utilize cellular nutrients in the early phases of infection [27]; however, in late infection phases, proliferating $T$. gondii suppress autophagy in host cells [28]. Recent studies suggest that canonical [29] or non-canonical autophagy $[30,31]$ are involved in the restriction of intracellular T. gondii.

Protozoan parasites of the phylum Apicomplexa can survive and proliferate within diverse cell types. They are the etiological agent for various human diseases including toxoplasmosis, malaria, and cryptosporidiosis. 4-HBA is a hydroxybenzalde- 
hyde and a major bioactive compound of Gastrodiae Rhizoma. A recent study suggests that 4-HBA, isolated from Alpinia conchigera, is one of the antimicrobials that affects Staphylococcus aureus, Microsporum canis, Candida albicans, and Trichophyton rubrum [32]. However, the roles of 4-HBA in autophagy and antiparasitic responses have not been investigated. In this study, we found that 4-HBA induced autophagy in primary macrophages, which is required to inhibit the intracellular growth of T. gondii.

To date, 7 mammalian Sirts have been identified. They are essential modulators of cell survival/death and energy status [33]. Among them, SIRT1, a class III histone deacetylase and $\mathrm{NAD}^{+}$-dependent protein, acts as a metabolic sensor of cellular energy. [19]. Several studies have demonstrated that SIRT1 activation is required for the regulation of various cellular responses [34]. For example, resveratrol, a SIRT1 activator, promotes SIRT1-dependent autophagy and ameliorates excessive inflammatory responses $[35,36]$. Moreover, we previously reported that the AMP-activated protein kinase pathway and SIRT1-induced orphan nuclear receptor estrogen-related receptor $\alpha$ (ESRRA) expression are required for the activation of autophagy and antimicrobial responses against Mycobacterium tuberculosis infection [37]. Herein, we found that the inactivation of SIRT1 inhibited 4-HBA-induced autophagy and antiparasitic responses, indicating that SIRT1-mediated autophagy is crucial for host-protective effects of 4-HBA against $T$. gondii infection.

In summary, we found that 4-HBA significantly restricted the intracellular growth of T. gondii via SIRT1-mediated autophagy in primary murine macrophages, and mediated $T$. gondii infection. These findings suggest that 4-HBA may be a promising, novel toxoplasmosis therapeutic. In future studies, we will evaluate the potential synergy between 4-HBA and standard therapeutics, such as pyrimethamine and sulfadiazine, to treat T. gondii infection.

\section{ACKNOWLEDGMENTS}

We thank Jaeyul Kwon and Guang-Ho Cha (Chungnam National University) for scientific and language editing. This research was supported by Basic Science Research Program through the National Research Foundation of Korea (NRF) funded by the Ministry of Science, ICT and Future Planning (NRF-2017R1A5A2015385, NRF-2019R1C1C1004431, and NRF2018M2A2B3A02072071).

\section{CONFLICT OF INTEREST}

The authors have no financial conflict of interests.

\section{REFERENCES}

1. Dubremetz JF. Host cell invasion by Toxoplasma gondii. Trends Microbiol 1998; 6: 27-30.

2. Elmore SA, Jones JL, Conrad PA, Patton S, Lindsay DS, Dubey JP, Toxoplasma gondii: epidemiology, feline clinical aspects, and prevention. Trends Parasitol 2010; 26: 190-196.

3. Dupont CD, Christian DA, Hunter CA. Immune response and immunopathology during toxoplasmosis. Semin Immunopathol 2012; 34: 793-813.

4. Derouin F, Anti-toxoplasmosis drugs. Curr Opin Investig Drugs 2001; 2: 1368-1374.

5. Klionsky DJ, Emr SD. Autophagy as a regulated pathway of cellular degradation. Science 2000; 290: 1717-1721.

6. Rabinowitz JD, White E. Autophagy and metabolism. Science 2010; 330: 1344-1348.

7. Levine B, Mizushima N, Virgin HW. Autophagy in immunity and inflammation. Nature 2011; 469: 323-335.

8. Skendros P, Mitroulis I. Host cell autophagy in immune response to zoonotic infections. Clin Dev Immuno 2012; 2012: 910525.

9. Besteiro $\mathrm{S}$. The role of host autophagy machinery in controlling Toxoplasma infection. Virulence 2019; 10: 438-447.

10. Park S, Kim DS, Kang S. Gastrodia elata Blume water extracts improve insulin resistance by decreasing body fat in diet-induced obese rats: vanillin and 4-hydroxybenzaldehyde are the bioactive candidates. Eur J Nutr 2011; 50: 107-118.

11. Ha JH, Lee DU, Lee JT, Kim JS, Yong CS, Kim JA, Ha JS, Huh K. 4-Hydroxybenzaldehyde from Gastrodia elata B1. is active in the antioxidation and GABAergic neuromodulation of the rat brain. J Ethnopharmacol 2000; 73: 329-333.

12. Kang CW, Han YE, Kim J, Oh JH, Cho YH, Lee EJ. 4-Hydroxybenzaldehyde accelerates acute wound healing through activation of focal adhesion signalling in keratinocytes. Sci Rep 2017; 7: 14192.

13. Kim WS, Song HY, Mushtaq S, Kim JM, Byun EH, Yuk JM, Byun EB. Therapeutic Potential of Gamma-Irradiated Resveratrol in Ulcerative Colitis via the Anti-Inflammatory Activity and Differentiation of Tolerogenic Dendritic Cells. Cell Physiol Biochem 2019; 52: 1117-1138.

14. Shin B, Park C, Imlay JA, Park W. 4-Hydroxybenzaldehyde sensitizes Acinetobacter baumannii to amphenicols. Appl Microbiol Biotechnol 2018; 102: 2323-2335.

15. Yuk JM, Shin DM, Lee HM, Kim JJ, Kim SW, Jin HS, Yang CS, Park KA, Chanda D, Kim DK, Huang SM, Lee SK, Lee CH, Kim JM, Song CH, Lee SY, Hur GM, Moore DD, Choi HS, Jo EK. The orphan nuclear receptor SHP acts as a negative regulator in inflammatory signaling triggered by Toll-like receptors. Nat Immu- 
nol 2011; 12: 742-751.

16. Yang CS, Kim JJ, Lee HM, Jin HS, Lee SH, Park JH, Kim SJ, Kim JM, Han YM, Lee MS, Kweon GR, Shong M, Jo EK. The AMPKPPARGC1A pathway is required for antimicrobial host defense through activation of autophagy. Autophagy 2014; 10: 785-802.

17. Yarovinsky F. Innate immunity to Toxoplasma gondii infection. Nat Rev Immunol 2014; 14: 109-121.

18. Zhu W, Li J, Pappoe F, Shen J, Yu L. Strategies Developed by Toxoplasma gondii to Survive in the Host. Front Microbiol 2019; 10: 899.

19. Canto C, Auwerx J. PGC-1alpha, SIRT1 and AMPK, an energy sensing network that controls energy expenditure. Curr Opin Lipidol 2009; 20: 98-105.

20. Yang T, Fu M, Pestell R, Sauve AA, SIRT1 and endocrine signaling. Trends Endocrinol Metab 2006; 17: 186-191.

21. Kong S, McBurney MW, Fang D. Sirtuin 1 in immune regulation and autoimmunity. Immunol Cell Biol 2012; 90: 6-13.

22. Ng F, Tang BL. Sirtuins' modulation of autophagy. J Cell Physiol 2013; 228: 2262-2270.

23. Thorburn A. Autophagy and disease. J Biol Chem 2018; 293 : 5425-5430.

24. Sil P, Muse G, Martinez J. A ravenous defense: canonical and noncanonical autophagy in immunity. Curr Opin Immunol. 2018; 50: 21-31.

25. Sharma V, Verma S, Seranova E, Sarkar S, Kumar D. Selective autophagy and xenophagy in infection and disease. Front Cell Dev Biol 2018; 6: 147.

26. Prieto-Dominguez N, Garcia-Mediavilla MV, Sanchez-Campos S, Mauriz JL, Gonzalez-Gallego J. Autophagy as a molecular target of flavonoids underlying their protective effects in human disease. Curr Med Chem 2018; 25: 814-838.

27. Wang Y, Weiss LM, Orlofsky A. Host cell autophagy is induced by Toxoplasma gondii and contributes to parasite growth. J Biol Chem 2009; 284: 1694-1701.

28. Lee YJ, Song HO, Lee YH, Ryu JS, Ahn MH. Proliferation of Toxoplasma gondii suppresses host cell autophagy. Korean J Parasitol 2013; 51: 279-287.
29. Choi J, Park S, Biering SB, Selleck E, Liu CY, Zhang X, Fujita N, Saitoh T, Akira S, Yoshimori T, Sibley LD, Hwang S, Virgin HW. The parasitophorous vacuole membrane of Toxoplasma gondii is targeted for disruption by ubiquitin-like conjugation systems of autophagy. Immunity 2014; 40: 924-935.

30. Selleck EM, Orchard RC, Lassen KG, Beatty WL, Xavier RJ, Levine B, Virgin HW, Sibley LD. A noncanonical autophagy pathway restricts Toxoplasma gondii growth in a strain-specific manner in IFN-gamma-activated human cells. MBio 2015; 6: e01157-15.

31. Leveque MF, Berry L, Cipriano MJ, Nguyen HM, Striepen B, Besteiro S. Autophagy-related protein ATG8 has a noncanonical function for apicoplast inheritance in Toxoplasma gondii. MBio 2015; 6: e01446-15.

32. Aziz AN, Ibrahim H, Rosmy Syamsir D, Mohtar M, Vejayan J, Awang K. Antimicrobial compounds from Alpinia conchigera. J Ethnopharmacol. 2013; 145: 798-802.

33. Houtkooper RH, Pirinen E, Auwerx J. Sirtuins as regulators of metabolism and healthspan. Nat Rev Mol Cell Biol 2012; 13: 225-238.

34. Chung S, Yao H, Caito S, Hwang JW, Arunachalam G, Rahman I. Regulation of SIRT1 in cellular functions: role of polyphenols. Arch Biochem Biophys 2010; 501: 79-90.

35. Yang QB, He YL, Zhong XW, Xie WG, Zhou JG. Resveratrol ameliorates gouty inflammation via upregulation of sirtuin 1 to promote autophagy in gout patients. Inflammopharmacology 2019; 27: 47-56.

36. Chen ML, Yi L, Jin X, Liang XY, Zhou Y, Zhang T, Xie Q, Zhou X, Chang H, Fu YJ, Zhu JD, Zhang QY, Mi MT. Resveratrol attenuates vascular endothelial inflammation by inducing autophagy through the cAMP signaling pathway. Autophagy 2013; 9: 20332045.

37. Kim SY, Yang CS, Lee HM, Kim JK, Kim YS, Kim YR, Kim JS, Kim TS, Yuk JM, Dufour CR, Lee SH, Kim JM, Choi HS, Giguere V, Jo EK. ESRRA (estrogen-related receptor alpha) is a key coordinator of transcriptional and post-translational activation of autophagy to promote innate host defense. Autophagy 2018; 14: 152-168. 